\title{
6.2 KOOPERATIVE „ENERGIEWENDE“?
}

Auf Kooperation als Prinzip Vorsorgenden Wirtschaftens wurde in Kapitel 3.2.1 bereits eingegangen. Im ökonomischen Handlungsraum bezeichnet kooperieren die gemeinsame Ausführung einer ökonomischen Tätigkeit (Biesecker/Kesting 2003: 180). Die Frage, wie diese Gemeinsamkeit gestaltet ist, hat jedoch großen Einfluss darauf, ob eine Kooperation tatsächlich als Kooperation im Sinne Vorsorgenden Wirtschaftens gelten kann oder nicht. Verschiedene Typen von Kooperation ${ }^{6}$ wurden in Kapitel 3.2.1 vorgestellt. Im Folgenden analysiere ich, welche Vorstellungen des wirtschaftlichen, sozialen und politischen Zusammenwirkens in den Debatten identifiziert werden können, ob diese eher konkurrenz-/wettbewerbs- oder kooperationsbasiert sind und auf welche Typen von Kooperation Bezug genommen wird. Außerdem wird das Einbeziehen der gesellschaftlichen Naturverhältnisse im Sinne der in Kapitel 3 ausgeführten Kategorie der (Re)Produktivität in den „Energiewende“ - Diskurs überprüft.

Auch das in seiner Wirkung als reformistisch klassifizierte Narrativ der ,ökologischen Modernisierung', das sich im Kontext der politischen Praktiken der rot-grünen Regierung in der 14. und 15. Legislaturperiode zeigt, setzt eher an einem konkurrenzund wettbewerbsbasierten als an einem kooperativen Ökonomieverständnis an. Zukünftige Generationen sowie ökologische Fragen werden zwar stärker als bisher in die politische Entscheidungsfindung eingebunden, auch sie werden aber marktökonomisch eingeordnet. Der Erhalt und die Verbesserung der Wettbewerbsfähigkeit sind stets ein Ziel umweltorientierter energiepolitischer Maßnahmen, ökologische Krisen werden zur Chance uminterpretiert, die Ökologie der Ökonomie als vorgelagert betrachtet: Durch die Bekämpfung des Klimawandels über die Förderung erneuerbarer Energien solle die internationale Wettbewerbsfähigkeit der deutschen Wirtschaft gesteigert werden (vgl. Kapitel 5.2.3). Über die Ökosteuer, deren Einnahmen nicht für ökologische Zwecke eingesetzt werden, sondern um die Lohnnebenkosten zu senken, solle die Wettbewerbsfähigkeit der Unternehmen erhöht werden (vgl. z.B. Koalitionsvertrag 1998: 12; vgl. Kapitel 5.2.1); Wettbewerb wird also als Ziel und Mittel politischer Praktiken zugleich formuliert. Dabei wird auch in dem zumindest in seinen Ansätzen auf Sorge für die zukünftige Gegenwart ,Anderer' ausgerichteten Narrativ der ,ökologischen Modernisierung‘ Natur nicht im Sinne von (Re)Produktivität als ,Kooperationspartnerin“ in ökonomisches Handeln mit einbezogen. Eine Vermittlung zwischen Arbeits- und Naturproduktivität (Biesecker/Hofmeister 2006; Biesecker/Hofmeister 2013a) ist nicht erkennbar.

Im Spannungsfeld zwischen Kooperation und marktwirtschaftlicher Konkurrenz stellen sich die Artikulationen zur Implementierung erneuerbarer Energien unter dem Narrativ der ,ökologischen Modernisierung' ambivalent dar. Einerseits ist die Artikulation ,erneuerbarer Energien' stark durch die Story-Line determiniert, welche die Marktwirtschaft als Bedingung für erneuerbare Energiepolitik konstituiert. Andererseits schlägt sich dies in den politischen Praktiken insofern zunächst nicht wieder, als dass das EEG im Kern nicht als (rein) marktwirtschaftliches Instrument angelegt wird, sondern über feste Einspeisevergütungen als Förderinstrument für erneuerbare 
Energien. Dass sich erneuerbare Energien im EEG nicht „,dem Wettbewerb [...] stellen müssten“ (CDU 25.02.2000: 8430), wird von CDU/CSU und FDP als Grund angeführt, dieses zunächst abzulehnen (vgl. Kapitel 5.2.4). Zwar wird die Bedeutung des EEGs als Markteinführungsinstrument betont, gleichzeitig argumentiert aber die SPD, dass erneuerbare Energien ,nicht nach dem traditionellen Bewertungsmuster von Wettbewerb eingeführt werden“ könnten (SPD 25.02.2000: 8439; vgl. Kapitel 5.2.4). Entsprechend materialisiert sich das EEG außerhalb der Bundestagsdebatten in Elementen verständiger Kooperation, indem es bspw. Privatpersonen ${ }^{7}$ und Bürger*innengenossenschaften, die auf demokratische Entscheidungsfindung ausgerichtet sind und nicht in erster Linie profitorientiert ${ }^{8}$ wirtschaften, Gestaltungsmacht zuweist und ihnen über feste Einspeisevergütungen die Teilhabe am Energieproduktionsprozess ermöglicht (Amri-Henkel/Hofmeister 2018). Gesellschaftliche Asymmetrien werden allerdings kaum benannt. In den Debatten um das EEG wird das Bild eines (männlichen) Eigenheimbesitzers (GRÜNE 29.03.2012: 20203) gezeichnet, dem die Rolle des Investors (SPD 25.02.2000: 8437) in erneuerbare Energien zugewiesen wird, während seine Funktion im gesellschaftlichen Gestaltungsprozess der „Energiewende" oder als möglicherweise aktive*r Konsument*in kaum bestimmt wird (vgl. Kapitel 5.2.8). Es erfolgt im politischen Diskurs hier also kaum eine Einbeziehung der „,menschlichen Tätigkeiten und [der] Naturleistungen [...] in das Ökonomische“ (Biesecker/Hofmeister 2013a: 154) im Sinne von (Re)Produktivität. Die Elemente verständiger und vorsorgend-verantwortlicher Kooperation, die durch das EEG ermöglicht werden, werden in den Debatten von der Regierungskoalition nicht angesprochen/explizit gemacht, während marktökonomische Legitimationen im Vordergrund stehen.

In der 16. Legislaturperiode nimmt die Ausrichtung des EEG auf Wettbewerb zu und die Vergütungssätze werden 2009 mit der Begründung der internationalen Wettbewerbsfähigkeit verringert - schließlich sei die ,,ausländische Konkurrenz [...] aus den Startblöcken heraus“ (vgl. CDU 06.06.2008: 17742; vgl. Kapitel 5.2.4). Energiepolitische Maßnahmen im Hinblick auf die im Koalitionsvertrag 2013 bemühte ,Nachhaltigkeit' werden auch in der 16. Legislaturperiode nur insofern als akzeptabel betrachtet, als sie die Wettbewerbsfähigkeit nicht einschränken und gleichzeitig einen ökonomischen Nutzen erbringen (vgl. z.B. BMU 2007: 4). So gelte es, die energieintensive Industrie durch Kostensteigerungen ,in ihrer internationalen Wettbewerbsfähigkeit nicht zu beeinträchtigen“ (Koalitionsvertrag 2005: 85).

Zukünftige Generationen als berechtigte Anspruchsgruppe und Kooperationspartnerinnen im Verständnis Vorsorgenden Wirtschaftens finden in den politischen Entscheidungsprozessen zwar vereinzelt Erwähnung, werden aber kaum berücksich-

7 Natürlich kann die Teilhabe von Privatpersonen am Energieproduktionsprozess auch strategisch motiviert, also eigennutzorientiert sein. Ich spreche hier von ,Elementen` verständiger Kooperation, da die Verschiebung von Machtasymmetrien durch das EEG die Teilhabe zuvor vom Produktionsprozess ausgeschlossener Personengruppen immerhin ermöglicht.

8 Eine ausschließliche Gewinnorientierung für Energiegenossenschaften ist in Deutschland durch das Genossenschaftsgesetz ausgeschlossen. Gewinn dient der Erfüllung des Förderauftrags nach $\S 1 \mathrm{GenG}$. 
tigt. Umweltpolitische Fragen bleiben marginal (vgl. Kapitel 5.1.2). Das Konkurrenzprinzip - auf volkswirtschaftlicher Ebene verkörpert im Wettbewerbsprinzip steht im Vordergrund politischer Handlungsorientierung. Asymmetrien (bspw. in Form zu hoher Strompreise durch Spekulation) zu Ungunsten der Verbraucher*innen werden abgestritten: Die Märkte seien „,von echten physischen Interessen [und nicht von Spekulation] getrieben“ (CDU 02.07.2009: 25871). Die LINKEN fordern eine demokratische Koordination, bzw. Kontrolle des Strommarktes durch eine Strompreisbehörde (LINKE 02.07.2009: 25876), was von den anderen Fraktionen überwiegend mit dem Argument des Wettbewerbs abgelehnt wird. So argumentieren die GRÜNEN eine solche Behörde sei durch ihre monopolartige Stellung ,der natürliche Feind des Fortschritts“ (GRÜNE 02.07.2009: 25877). Sie fordern stattdessen „,fairen Wettbewerb“ (GRÜNE 02.07.2009: 25877) (vgl. Kapitel 5.2.4).

Zivilgesellschaftliche Akteur*innen werden in den Entscheidungsprozess der Laufzeitverlängerung der Atomkraftwerke in der 17. Legislaturperiode trotz zahlreicher Demonstrationen ${ }^{9}$ nicht nur nicht eingebunden, sondern größtenteils ignoriert (sie werden lediglich von der Opposition erwähnt). ${ }^{10}$ Gleichzeitig wird im Energiekonzept eine aktivere Beteiligung innerhalb eines Dialogforums „nachhaltige Energieversorgung" für die Zukunft in Aussicht gestellt, um den energiepolitischen Konsens zu ,verbreitern“ (17/3049: 18). Dabei werden die Konflikthaftigkeit des Energiekonzeptes und die breite gesellschaftliche Ablehnung der Kernenergie verdeckt, indem eine generelle Kooperationsbereitschaft der Bundesregierung suggeriert wird. Im Gegensatz zu zivilgesellschaftlichen Akteur*innen werden die Energieversorgungsunternehmen aktiv in die Vereinbarungen zur Laufzeitverlängerung und zur Kernbrennstoffsteuer einbezogen, die zwischen der Bundesregierung und den Unternehmen im Vorfeld in zunächst geheimen Verträgen getroffen werden (vgl. z.B. GRÜNE 28.10.2010: 7160f.; LINKE 28.10.2010: 7175; GRÜNE 28.10.2010: 7189). ${ }^{11}$ In diesem Vorgehen erscheinen die Profitinteressen der Unternehmen als berechtigt - im Gegensatz zu nichtökonomischen Interessen anderer Anspruchsgruppen oder Interessen von kleineren Unternehmen wie Stadtwerken. Auch durch das NichtEinbeziehen zivilgesellschaftlicher Akteur*innen und bspw. Umweltverbänden entfällt eine Vermittlung zwischen sozial-ökologischen Belangen und dem Ökonomischen im Sinne von (Re)Produktivität. Als legitime Interessen im Entscheidungspro-

9 Beispielsweise demonstrieren im September 2010 zwischen 40.000 und 100.000 Menschen in Berlin gegen die Laufzeitverlängerung (www.zeit.de - Proteste).

10 Die Mehrheit der deutschen Bevölkerung ist zu diesem Zeitpunkt laut einer Umfrage von TNS Emnid im Auftrag der ZEIT gegen eine Laufzeitverlängerung von mehr als 10 Jahren. So lehnten 48 Prozent der Deutschen eine Verlängerung der Laufzeiten der Kernkraftwerke generell ab, 29 Prozent wollen eine Verlängerung um höchstens 10 Jahre (www.zeit.de Laufzeitverlängerung).

11 Zudem kritisiert die Opposition, die Bundesregierung habe für ihre Interessenvertretung in den Verhandlungen einen Atomkraftlobbyisten beauftragt: „Sie, Umweltminister Röttgen, lagen in der Nacht, in der die Vereinbarung mit den Konzernen unterschrieben wurde, im Bett. Die Feder der Regierung führte der bekennende Atomlobbyist Hennenhöfer. Freunde unter sich beim Aushandeln der Revolution zurück ins 20. Jahrhundert!“ (GRÜNE 28.10.2010: 7189). 
zess zur Laufzeitverlängerung konstituiert die Regierungskoalition ausschließlich wirtschaftliche Interessen, deren Berücksichtigung als Sachzwang dargestellt wird bspw. der Erhalt von Arbeitsplätzen im konventionellen Energiebereich (vgl. Kapitel 5.2.1). Andere politische Positionen gegen Atomenergie werden als illegitim konstituiert, die Verweigerung der Kooperation durch die Opposition als „Zukunftsverweigerung und Verantwortungsverweigerung“ (CDU 28.10.2010: 7179). Die Kooperation, wie sie von der schwarz-gelben Koalition eingefordert wird, wird demnach nicht mit einer zivilgesellschaftlichen Teilhabe an Entscheidungsprozessen verbunden, ist also nicht als gleichberechtigte Kooperation zu verstehen, sie basiert auf einem herrschaftlich-autoritären Anspruch, der sich aus einer Unterordnung unter eine naturalisierte marktwirtschaftliche Ideologie ableitet.

Das Ereignis „Fukushima“ holt die nichtmarktlichen Akteur*innen insofern in den Blick, als sich die Vorbehalte zahlreicher zivilgesellschaftlicher Akteur*innen gegenüber der Atomenergie als berechtigt erweisen. Die Regierungskoalition versucht nun die nichtmarktlichen, zivilgesellschaftlichen Akteur*innen in einen neuen vermeintlich kooperativen Konsens mit einzubeziehen. In einem ersten Schritt wird die „Ethikkommission Sichere Energieversorgung“ einberufen, als deren Aufgabe es beschrieben wird, ,die Energiewende auf einen breiten gesellschaftlichen Konsens zu stellen" (www.bundesregierung.de - Ethikkommission). Mitglied sind überwiegend Vertreter*innen aus Wissenschaft und Politik, aber auch aus Wirtschaft, Gewerkschaft und Kirche. Weitere Interessensgruppen (z.B. Umweltverbände, Wirtschaftsverbände, Energieversorgungsunternehmen, Jugendverbände) werden im Rahmen der Kommissionsarbeit angehört. Eine über die Arbeit in der Kommission hinausgehende Einbindung von Akteur*innen in Entscheidungsprozesse wird allerdings kaum diskutiert und lediglich von der Opposition eingefordert. ${ }^{12}$ Bürger*innen spielen in den Bundestagsdebatten um die „Energiewende“ weiterhin eine untergeordnete Rolle (vgl. Kapitel 5.1.2), ihrer Einbindung scheint mit Abschluss der Kommission, deren Diskussion sich in den Bundestagsdebatten überwiegend auf den Atomausstieg beschränkt, genüge getan. Gleichzeitig wird die Gesamtgesellschaft sprachlich in den ,neuen“ Konsens eines kollektiven „Wir“ einbezogen, verbunden mit dem Appell, zukünftigen Protest zu unterlassen (Angela Merkel 09.06.2011: 12961). So soll nicht nur der Eindruck geweckt werden, die Regierungskoalition teile das Anliegen einer „Energiewende“ mit den Bürger*innen und anderen zivilgesellschaftlichen Akteur*innen, es wird auch eine verständigungsorientierte Kooperation mit allen gesellschaftlichen Gruppen suggeriert. Dieser ist aber ein herrschaftlich-autoritärer Charakter zu attestieren, da unterschiedliche Standpunkte nicht anerkannt bzw. abgestritten werden und eine politische Auseinandersetzung darüber verweigert wird. ${ }^{13}$

Eine Vermittlung zwischen Arbeits- und Naturproduktivität findet auch nach „Fukushima“ nicht statt, da die Regierungskoalition die Artikulation des Katastrophenszenarios einer „Deindustrialisierung“ aufrechterhält (FDP 24.03.2011: 11286) und so weder die sozial-ökologische Krise noch potenziell transformative sozial-

12 So fordern die LINKEN die kooperative Erarbeitung eines neuen Energiekonzeptes, in das verschiedene Interessensgruppen einbezogen werden (LINKE 17.03.2011: 10898).

13 Vgl. z.B. die Aussage der CDU, dass ,alle Streitigkeiten [...] in den Grundfragen“ damit beseitigt seien (CDU 30.06.2011: 13369). 
ökologische Arrangements in den Blick kommen (vgl. Kapitel 5.4). Das EEG wird weiter sozial-ökologisch entbettet, indem es ,so wirtschaftsfreundlich und industriefreundlich“ gemacht werden solle, ,wie es noch nie war“ (CDU 30.06.2011: 13370), während sozial-ökologische Sichtweisen ausgeblendet werden (vgl. Kapitel 5.4). Die Elemente verständiger und vorsorgend-verantwortlicher Kooperation, die durch das EEG ermöglicht wurden und stets im Schatten der Debatten verblieben, werden nun zunehmend zurückgedrängt. ${ }^{14}$ Durch die Novellierungen des EEG 2014 und 2016/17 und dem damit verbunden „Systemwechsel hin zu Ausschreibungen“ (CDU 01.06.2016: 16984) wird das Konkurrenzprinzip als handlungskoordinierendes Prinzip der „Energiewende“ weiter gestärkt (vgl. Kapitel 5.2.4). Preise sollen künftig durch Wettbewerb und nicht mehr durch das Gesetz festgelegt werden. Zugrunde liegt die Annahme, dass die Teilnehmenden eines solchen Bieterverfahrens rational und eigennutzorientiert handeln. Für Bürger*innenenergiegesellschaften werden diese ökonomischen Prämissen genauso vorausgesetzt wie für Energieversorgungsunternehmen. Asymmetrien werden zwar insofern berücksichtigt, dass für Bürger*innenenergiegesellschaften besondere Bedingungen gelten. Die Teilnahme am Bieterverfahren erfordert aber ein hohes $\mathrm{Ma} ß$ an strategischem und marktfokussiertem Handeln, im Gegensatz zu kommunikativem oder vorsorgendem Handeln (vgl. Biesecker/Kesting 2003: 177) und ist daher für profitorientierte Unternehmen attraktiver als für größtenteils ehrenamtlich arbeitende Bürger*innenenergiegesellschaften. Strategische Akteur*innen, die auf Grundlage einer eigennutzorientierten ökonomischen Rationalität arbeiten sind daher im Vorteil - kooperative Eigentumsformen werden zurückgedrängt. ${ }^{15}$ Es kommt zu einer Machtverschiebung von herkömmli-

14 Die neuen strukturellen Rahmenbedingungen begünstigen eher eine strategische Handlungsorientierungen, da Anlagenbetreiber im Gegensatz zur bisherigen festen Einspeisevergütung nun als aktive Marktakteur*innen im Wettbewerb gesehen werden, die durch die sich nach dem Börsenstrompreis richtende „Marktprämie“ auf Preissignale reagieren sollen. Dass dies bei fluktuierenden erneuerbaren Energien überhaupt nicht möglich ist, solange keine Speichermöglichkeiten bestehen, wird dabei ebenso ausgeblendet wie die Tatsache, dass das Strommarktmodell, welches den Börsenstrompreis bestimmt, auf fossile Energien ausgerichtet ist (Schomerus/Henkel 2012: 19).

15 Diese Einschätzung bestätigt sich bei einer genaueren Betrachtung der Zahlen aus der Datenbank für Bürgerenergie, die an der Leuphana Universität Lüneburg geführt wird. So zeigt sich zwischen 2009 und 2013 ein Aufschwung in den Neugründungen von Bürger*innenenergiegesellschaften (darunter vor allem auch Genossenschaften), der hauptsächlich den rechtlichen Rahmenbedingungen des EEG geschuldet ist. Seit 2014 ist die Anzahl der neu gegründeten Energiegenossenschaften jedoch deutlich gesunken, die Anzahl an Löschungen angestiegen. Gleichzeitig sind die Neugründungen von Bürger*innenenergiegesellschaften in anderen Rechtsformen wie der GmbH \& Co KG gestiegen. Dies liegt auch an einer Verschiebung der EEG-Förderung weg von PV hin zu Windenergie. Es kam zu deutlichen Verschiebungen in der Zusammensetzungen des Bürger*innenenergiesektors (Kahla u.a. 2017: 13ff.). Kahla u.a. (2017) beschreiben den Unterschied zwischen den beiden Rechtsformen im Bürger*innenenergiebereich wie folgt: „(Viele) Energiegenossenschaften entstehen trotz der vorhandenen Gründungsunterstützungsstrukturen eher emergent; d.h. die Gründungsprozesse von Energiegenossenschaf- 
chen größtenteils auf Privatpersonen basierenden Bürger*innenenergiegesellschaften zu strategischen, von Unternehmen initiierten, vermeintlichen Bürger*innenenergiegesellschaften (vgl. Kapitel 5.2.8).

Insgesamt werden in den Debatten um die „Energiewende“ sowie erneuerbare Energien kooperative Vorstellungen des Zusammenwirkens marginalisiert und die Gestaltung der Energiepolitik marktwirtschaftlichen Prinzipien unterworfen. Machtasymmetrien im Wettbewerb werden bis auf wenige Ausnahmen (bspw. der Verweis auf die Macht der , großen Vier', vor allem durch SPD, GRÜNE und LINKE) nicht artikuliert. Es dominiert die implizite Annahme von gleichen Ausgangsbedingungen, eines Wettbewerbs unter Gleichen. Normative, sozial-ökologische Handlungsorientierungen geraten aus dem Blick. Dies führt zu einer diskursiven Verfestigung der gesellschaftlichen Naturverhältnisse, in denen die Natur der Wettbewerbslogik unterworfen wird (Krüger 2015: 101; Görg 2003: 140). ${ }^{16}$ Natur wird somit nicht als gleichberechtigte Akteurin in einem ökonomischen Kooperationsprozess gefasst, sondern wird nutzenorientiert angeeignet und als Mittel zum Zweck betrachtet. Arbeit wird nur im Sinne von Erwerbsarbeit adressiert, Sorgearbeit ist kein Gegenstand der Debatten. Kooperative, sozial-ökologische, geschweige denn (re)produktive politische Praktiken werden so verunmöglicht.

\subsection{AM GUTEN LEBEN ORIENTIERTE „ENERGIEWENDE“?}

In Kapitel 3.2.1 wurde Orientierung am für das gute Leben Notwendigen als handlungsleitendes Prinzip einer vorsorgenden Wirtschaftsweise eingeführt und zur Orientierung an monetären, rein marktorientierten Prinzipien abgegrenzt. Im Folgenden analysiere ich, welche handlungsleitenden Prinzipien sich in den untersuchten Debatten um Energiepolitik identifizieren lassen und welche Zielsetzungen und Wohlstandsvorstellungen damit verbunden werden.

Ein handlungsleitendes Prinzip des Narrativs der ,ökologischen Modernisierung‘ der 14. und 15. Legislaturperiode ist nicht unbedingtes Wachstum um jeden Preis, sondern ein Wachstum, welches mit ökologischen Zielvorstellungen vereinbar sein soll. Neben ökonomischen Zielen kommt auch der Erhalt der natürlichen Lebensgrundlagen als Lebensbedürfnis in den Blick. Reine Gewinnmaximierung wird nicht zur alleinigen Zielsetzung ökologischer Modernisierung erklärt: So werden überwiegend profitorientierte Motivationen wie bspw. der ,großen Vier‘ auch von der Regie-

ten sind im Vergleich zu den übrigen Bürgerenergiegesellschaften als eher nicht zentral gesteuert zu bezeichnen. Im Fall von GmbH und Co. KGs stehen meist Initiatoren in Form von Projektentwicklern, Flächeninhabern oder Ankerinvestoren bereit, die den Gründungsprozess der Bürgerenergiegesellschaft strukturieren. Im Gegensatz dazu sind in Energiegenossenschaften ehrenamtliche Gremienstrukturen anzutreffen, deren Motivation zu einem Großteil durch Altruismus erklärt werden muss“ (Kahla u.a. 2017: 25).

16 Auch wenn die oben dargestellten Effekte des EEG für verständige und vorsorgendverantwortlicher Kooperation außerhalb des Parlaments zum Teil andere gesellschaftliche Naturverhältnisse ermöglichen oder aufscheinen lassen (bspw. in Energiegenossenschaften, vgl. Amri-Henkel/Hofmeister (2018)). 